\title{
Anti-angiogenic effects of resveratrol in combination with 5-fluorouracil on B16 murine melanoma cells
}

\author{
SOL HWA LEE, BONG SEONG KOO, SONG YI PARK and YOUNG MIN KIM \\ Department of Biological Sciences and Biotechnology, College of Life Science and Nanotechnology, \\ Hannam University, Yusung-gu, Daejeon 305-811, Republic of Korea
}

Received January 2, 2014; Accepted October 24, 2014

DOI: $10.3892 / \mathrm{mmr} .2015 .3675$

\begin{abstract}
Fluorouracil (5-FU) has been used as a chemotherapeutic drug for various types of cancer, although the development of resistance remains a major limitation for its use in clinical settings. In the present study, the anti-angiogenic effects of resveratrol and 5-FU either alone or in combination were examined in a B16 murine melanoma model. Co-treatment using resveratrol and 5-FU inhibited cell proliferation more efficiently compared with use of either drug alone and the antiproliferative effect coincided with changes in the expression levels of AMP-activated protein kinase (AMPK), cyclooxygenase-2, vasodilator-stimulated phosphoprotein (VASP) and vascular endothelial growth factor (VEGF). Furthermore, co-treatment with resveratrol and 5-FU reduced tumor growth compared with that in the control group and this growth-inhibitory effect was associated with changes in the expression levels of AMPK, VASP and VEGF. Immunohistochemical staining for angiogenesis demonstrated that co-treatment with resveratrol and 5-FU reduced the number of microvascular vessels compared with that in the control group. These results suggested that co-treatment with resveratrol and 5-FU suppressed cell growth and angiogenesis in B16 murine melanoma tumors.
\end{abstract}

\section{Introduction}

Resveratrol, a natural polyphenol found in grapes and red wine, is known to have various effects on antioxidant activity (1) and can induce apoptosis and inhibit angiogenesis in various types of cancer $(2,3)$. In previous studies, resveratrol has been observed to induce apoptosis in glioma cells (4) and to inhibit the expression of hypoxia-inducible factor $1 \alpha$ and vascular

Correspondence to: Professor Young Min Kim, Department of Biological Sciences and Biotechnology, College of Life Science and Nanotechnology, Hannam University, Yuseong-daero 1646, Yuseong-gu, Daejeon 305-811, Republic of Korea

E-mail: kym@hnu.kr

Key words: resveratrol, 5-fluorouracil, angiogenesis, cyclooxygenase-2, vascular endothelial growth factor endothelial growth factor (VEGF) via multiple mechanisms, including inhibition of protein kinase $\mathrm{B}$ and mitogen-activated protein kinase (MAPK) in human ovarian cancer cell lines (5). In addition, resveratrol inhibits tumor growth and decreases angiogenesis by suppressing capillary-like tube formation by human umbilical vein endothelial cells in mouse models of lung cancer (6). Its potential as an antiangiogenic agent is further supported by its dose-dependent inhibition of tumor-induced neovascularization (7).

5-Fluorouracil (5-FU), a drug that induces apoptosis by inhibiting thymidylate synthase, has been used therapeutically in various types of cancer (8). However, its long-term use leads to resistance, limiting its clinical use (9). To overcome drug resistance in tumor cells, several studies have examined novel treatments or combination therapies with 5-FU. Previous studies have demonstrated that co-treatment with genistein and 5-FU induces apoptosis more efficiently compared with either drug alone in colon cancer and co-treatment with 5-FU and resveratrol is more efficient compared with controls in suppressing tumor cell growth in a murine model of liver cancer $(4,10)$.

Cyclooxygenase-2 (COX-2) is an inducible enzyme, which catalyzes the synthesis of prostaglandin $\mathrm{E}_{2}\left(\mathrm{PGE}_{2}\right)(11)$. The $\mathrm{COX}-2 / \mathrm{PGE}_{2}$ pathway is important in inflammation, angiogenesis and tumorigenesis and its overexpression is found in various cancer cell lines $(12,13)$. It has been observed that downregulation of COX-2 suppresses angiogenesis and tumor growth via regulation of VEGF, angiopoietin-1, tie-2 and matrix metalloproteinase-2 (MMP2) in an in vivo model of gastric cancer (14). Furthermore, COX-2 binds directly to resveratrol and controls $\mathrm{PGE}_{2}$ and this complex inhibits the proliferation of colon cancer cells (15).

VEGF is overexpressed in a variety of cancer cells and is considered an important angiogenic factor, which is upregulated by hypoxia inducible factor-1 (16). Hypoxia-simulated VEGF regulates proliferation, migration and vascular permeability via various signaling pathways, including MAPK, phosphoinositide 3-kinase and protein kinase $\mathrm{C}$, in endothelial cells (17). Previous studies have demonstrated that VEGF-C-knockdown decreases proliferation and actin-mediated stress fiber formation in endothelial cells through the RhoA pathway (18) and that inhibition of the expression of VEGF suppresses tumor growth and vessel density in vivo (19).

Vasodilator-stimulated phosphoprotein (VASP) is expressed in vascular endothelial cells and smooth muscle 
cells and is important in the formation of endothelial cell substrates and contacts between cells by binding to actin, profilin, zyxin and vinculin (20). VASP regulates carcinoma cell invasion and metastasis in vivo and in vitro via epidermal growth factor (EGF) signaling (21).

The present study investigated the effect of resveratrol on the proliferation and metastatic potential of cancer cells via multiple pathways. In addition, the effect of combined treatment of resveratrol and 5-FU on the expression of VEGF, COX-2 and VASP as well as cell growth and migration was assessed on B16 melanoma cells. The present study indicated that co-treatment of melanoma cells with resveratrol and 5-FU was more efficient compared with either drug alone.

\section{Materials and methods}

Cells and reagents. B16 murine melanoma cells were purchased from the American Type Culture Collection (Manassas, VA, USA) and cultured in RPMI-1640 with 10\% fetal bovine serum (Gibco-BRL, Grand Island, NY, USA) at $37^{\circ} \mathrm{C}$ in a $5 \% \mathrm{CO}_{2}$ atmosphere. Resveratrol and 5-FU were purchased from Sigma (St. Louis, MO, USA). MTT and celecoxib were also obtained from Sigma. Monoclonal antibodies specific for phosphorylated (p)-AMP-activated protein kinase [AMPK; monoclonal rabbit immunoglobulin $\mathrm{G}$ (IgG)], COX-2 (polyclonal rabbit IgG), VASP (polyclonal rabbit IgG) and $\beta$-actin (polyclonal rabbit $\mathrm{IgG}$ ) were purchased from Cell Signaling Technology, Inc. (Beverly, MA, USA) and the VEGF (monoclonal mouse IgG) antibody was obtained from Santa Cruz Biotechnology, Inc. (San Diego, CA, USA).

Cell proliferation measurements. Cell proliferation was assessed using an MTT assay. The cells were seeded into a 12 -well plate $\left(1 \times 10^{6}\right.$ cells/well $)$ for $24 \mathrm{~h}$ and were incubated with resveratrol $(10-50 \mu \mathrm{M})$ and 5-FU $(5-20 \mu \mathrm{M})$ for $24 \mathrm{~h}$. Following incubation with the test compounds, the cells were incubated with $30 \mu \mathrm{l}$ MTT solution $(5 \mathrm{mg} / \mathrm{ml})$ in phosphate-buffered saline (PBS) for $1 \mathrm{~h}$. The sample was then solubilized in dimethyl sulfoxide and the absorbance of purple formazan dye, the product of MTT converted by the viable cells, was quantified at $565 \mathrm{~nm}$ (Microplate Reader; Bio-Rad Laboratories, Inc., Hercules, CA, USA).

Detection of apoptosis. Following stimulation of the B16 murine melanoma cells with resveratrol and 5-FU, apoptosis was measured using a fluorescein isothiocyanate (FITC)-Annexin V apoptosis detectionkit(BDPharmingen,San Diego, CA, USA). The B16 cells, including floating cells, were collected following trypsinization (Trypsin-EDTA; Welegene, Inc., Deagu, Korea). The trypsinized cells were washed with PBS and resuspended in a mixture containing $100 \mu \mathrm{l}$ Annexin binding buffer mixed and $5 \mu$ l FITC-conjugated Annexin V and phycoerythrin-conjugated propidium iodide (PI). The resuspended cells were then incubated in the dark at room temperature for $15 \mathrm{~min}$, followed by analysis of the labeled cells by flow cytometry (FACS Calibur; Becton-Dickinson, Franklin Lakes, NJ, USA).

Wound healing migration assay. The B16 murine melanoma cells $\left(1 \times 10^{5}\right.$ cells $\left./ \mathrm{ml}\right)$ were seeded into a six-well plate for $24 \mathrm{~h}$.
The confluent monolayer was starved using serum-free medium for $12 \mathrm{~h}$ and wounded by scratching with a $200 \mu \mathrm{l}$ pipette tip (Axygen, Union City, CA, USA). The cells were incubated in serum-free medium containing the test compound at $37^{\circ} \mathrm{C}$ and images of the movement of cells into the wound area were captured at 0,24 and $48 \mathrm{~h}$ using a microscope (magnification, x100; CK40-F200, Olympus Corp., Tokyo, Japan).

Western blot analysis. The cells $\left(1 \times 10^{6}\right.$ cells $\left./ \mathrm{ml}\right)$ were incubated with resveratrol $(10-50 \mu \mathrm{M})$ and 5-FU $(5-20 \mu \mathrm{M})$ for $6 \mathrm{~h}$ on a six-well plate. The total proteins were extracted using radioimmunoprecipitation assay lysis buffer containing $50 \mathrm{mM}$ Tris- $\mathrm{HCl}$ (pH 8.0), 1\% NP 40, 0.5\% sodium deoxycholate, $150 \mathrm{mM} \mathrm{NaCl}$ and $1 \mathrm{mM}$ phenylmethylsulfonyl fluoride. The mixture was centrifuged at $19,326 x \mathrm{x}$ for $20 \mathrm{~min}$ at $4^{\circ} \mathrm{C}$ and the protein concentration was measured using a Bradford protein assay kit (Bio-Rad Laboratories, Inc.). Equal quantities of the protein were separated by SDS-PAGE and were then electrophoretically transferred onto nitrocellulose transfer membranes (Whatman, Pittsburgh, PA, USA). Following inhibition (BSA; Bovogen Biologicals Pty, Ltd, VIC, Australia), the membranes were incubated with phospho-AMPK $(1: 1,000)$, COX-2 (1:1,000), VASP $(1: 1,000), \beta$-actin $(1: 1,000)$ (Cell Signaling Technology, Inc.) and VEGF (1:1,000; Santa Cruz Biotechnology, Inc.) antibodies overnight at $4{ }^{\circ} \mathrm{C}$ with gentle agitation. Following incubation with the primary antibodies, the membrane was incubated with anti-mouse or anti-rabbit IgG secondary antibodies (Enzo Life Sciences, Farmingdale, $\mathrm{NY}$, USA) for $1.5 \mathrm{~h}$ at room temperature with gentle agitation. Following washing with TBST containing $20 \mathrm{mM}$ Tris, $500 \mathrm{mM}$ NaCL, pH 7.4 and $1 \mathrm{ml}$ Tween20 (Bio-Rad Laboratories, Inc.), the bands were visualized (Autoradiography cassette; Fisher Scientific, Inc., Pittsburgh, PA, USA) using enhanced chemiluminescence detection reagents (WEST-ZOL; iNtRON Biotechnology, Inc., Seoul, Korea).

Tumor formation. Male five-week-old Balb/c nu/nu mice were obtained from SLC, Inc. (Tokyo, Japan) and housed in sterile filer-topped cages. Mice were kept in an air-conditioned barrier facility at an ambient temperature of $25 \pm 2^{\circ} \mathrm{C}$, a relative humidity of $50 \pm 5 \%$ and a 12 -h on/off light cycle. Diets were purchased from Dyets Inc. (Bethlehem, PA, USA; AIN-76A Rodent Purified diet). Health was monitored daily by gross observation. For tumor induction, the B16 murine melanoma cells $\left(2.5 \times 10^{5}\right.$ cells $\left./ 0.1 \mathrm{ml}\right)$ were subcutaneously injected into the left flank of the mice ( $n=5 /$ group). Subsequently, one week after injection of the B16 murine melanoma cells, co-treatment with resveratrol and 5-FU was performed via injection into the left flank of Balb/c nu/nu mice for 10 days. The control animals were injected with a vehicle of PBS alone. The tumor size was measured using calipers at two-day intervals and the tumor volume was calculated using the modified formula $\mathrm{V}=1 / 2$ (length $\mathrm{x}$ width ${ }^{2}$ ). After 10 days of treatment, the tumors were excised and either frozen in liquid nitrogen for western blot analysis or fixed with formalin for immunohistochemical analysis. All surgery was performed under ether anesthesia, and efforts were made to minimize suffering. All animal experiments were approved by the Ethics Committee for Animal Experimentation, Hannam National University (Hannam, Korea). 
A

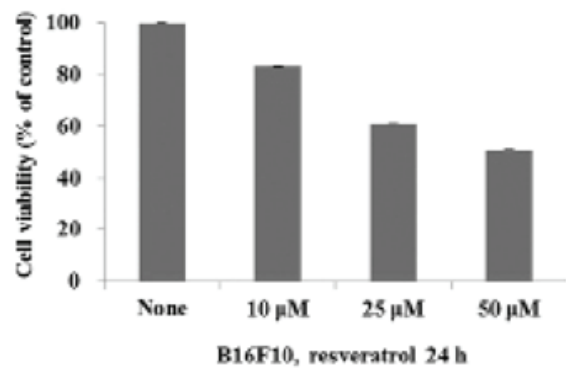

C

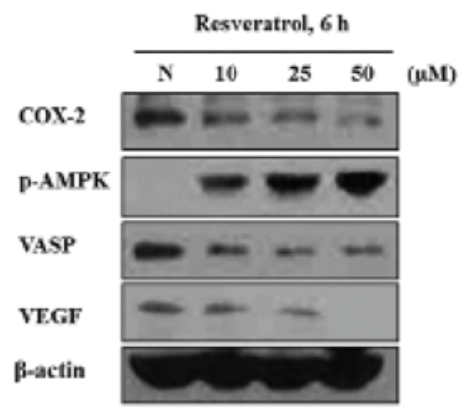

B

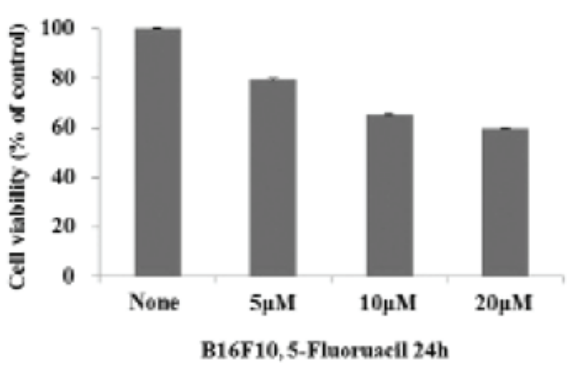

D

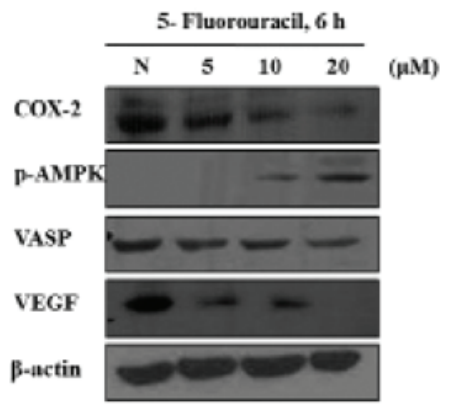

Figure 1. Resveratrol and 5-FU inhibit cell proliferation and regulate the expression levels of COX-2, VEGF and VASP in B16 cells. (A) Cells were treated with resveratrol $(10-50 \mu \mathrm{M})$ for $24 \mathrm{~h}$ and the cell viability was measured using an MTT assay. (B) Cells were treated with 5-FU (5-20 $\mu \mathrm{M})$ for $24 \mathrm{~h}$ and cell viability was measured using an MTT assay. (C) Cells were treated with resveratrol (10-50 $\mu \mathrm{M})$ for $6 \mathrm{~h}$ and total protein was subjected to western blot analysis using p-AMPK, COX-2, VEGF, VASP and $\beta$-actin (loading control) antibodies. (D) Cells were treated with 5-FU (5-20 $\mu \mathrm{M})$ for $6 \mathrm{~h}$ and total protein was subjected to western blot analysis using p-AMPK, COX-2, VEGF, VASP and $\beta$-actin (loading control) antibodies. p-AMPK, phosphorylated AMP-activated protein kinase; COX-2, cyclooxygenase-2; VASP, vasodilator-stimulated phosphoprotein; VEGF, vascular endothelial growth factor; FU, fluorouracil; N, none.

Immunohistochemistry. The tumor specimens from the mice were fixed in $10 \%$ formaldehyde, embedded in paraffin and sectioned into 5- $\mu \mathrm{m}$ slices. Consecutive 5- $\mu \mathrm{m}$ cryosections of optimum cutting temperature compound-embedded (Sakura Finetek, Torrance, CA, USA) tumor tissues were fixed in acetone at $4^{\circ} \mathrm{C}$ for $10 \mathrm{~min}$. Following washing in PBS, the sections were treated with $3 \% \mathrm{H}_{2} \mathrm{O}_{2}$ for 10 min to inhibit endogenous peroxidase activity and the sections were inhibited with normal rabbit serum. The sections were then washed in PBS and incubated with anti-CD31 antibody (rat-anti-mouse; Santa Cruz Biotechnology, Inc.) overnight at $4^{\circ} \mathrm{C}$. Negative controls were incubated with the primary normal serum immunoglobulin $\mathrm{G}$ for the species from which the primary antibody was obtained. The number of CD31-stained sites in the B16 murine melanoma tissues were counted, which corresponded to the microvessel density.

Statistical analysis. The microvessel density data were statistically analyzed using Student's t-test using SPSS 20 software (IBM SPSS, Armonk, NY, USA). Data are presented as the mean \pm standard deviation. $\mathrm{P}<0.05$ was considered to indicate a statistically significant difference.

\section{Results}

Resveratrol and 5-FU inhibit cell proliferation via the regulation of levels of AMPK, COX-2, VASP and VEGF in $B 16$ murine melanoma cells. To examine whether resveratrol and 5-FU exerted antiproliferative activity, the present study examined the effects of resveratrol and 5-FU on the growth of the B16 cells. The cells were treated with different concentrations $(10,25$ and $50 \mu \mathrm{M})$ of resveratrol for $24 \mathrm{~h}$ and the cell viability was evaluated using an MTT assay. As shown in Fig. 1A, the cell viability at concentrations of 10,25 and $50 \mu \mathrm{M}$ were 85,60 and $50 \%$, respectively. The cells were also treated with 5, 10 and $20 \mu \mathrm{M} \mathrm{5-FU}$ for $24 \mathrm{~h}$, resulting in a cell viability of 79,70 and $60 \%$, respectively (Fig. 1B). To investigate changes in the expression levels of AMPK, COX-2, VASP and VEGF, the B16 cells were treated with resveratrol and 5-FU for $6 \mathrm{~h}$ and the proteins were analyzed using western blot analysis. Resveratrol (Fig. 1C) and 5-FU (Fig. 1D) activated AMPK in a dose-dependent manner and decreased the expression levels of COX-2, VASP and VEGF, also in a dose-dependent manner.

Growth-inhibitory effects of combined resveratrol and 5-FU treatment on B16 cells are based on regulation of $A M P K, C O X-2$ VASP and VEGF. To investigate the effects of co-treatment with resveratrol and 5-FU on cell growth, B16 cells were treated with resveratrol and 5-FU either alone or in combination and the cell viability was measured using an MTT assay. When the cells were treated with $25 \mu \mathrm{M}$ resveratrol, $20 \mu \mathrm{M} 5$-FU or $25 \mu \mathrm{M}$ resveratrol in combination with $20 \mu \mathrm{M} 5-\mathrm{FU}$, the viability of the cells was 51,45 and $37 \%$, respectively (Fig. 2A). Thus, co-treatment with resveratrol and 5 -FU inhibited cell growth more efficiently compared with either resveratrol or 5-FU alone. To understand whether resveratrol- and 5-FU-induced cell death are mediated by apoptosis or necrosis, the apoptotic cell death was measured using Annexin V/PI staining. As Fig. 1B shows, $25 \mu \mathrm{M}$ resveratrol and $20 \mu \mathrm{M}$ 5-FU alone induced apoptosis; however, in combination, apoptosis was induced more efficiently. To determine 


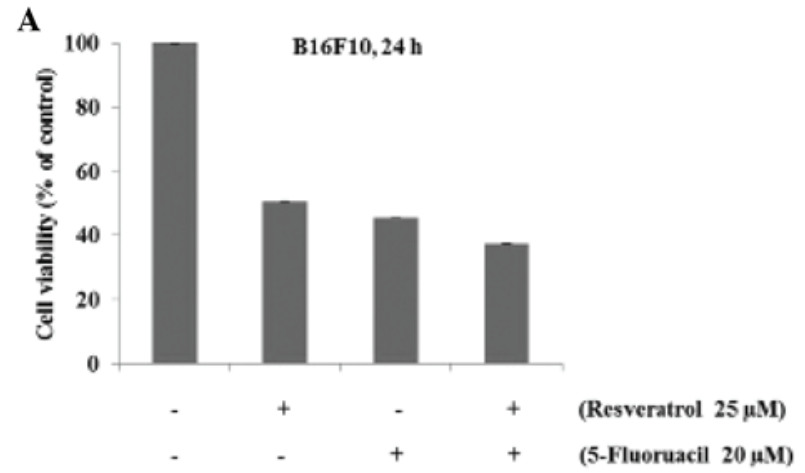

B

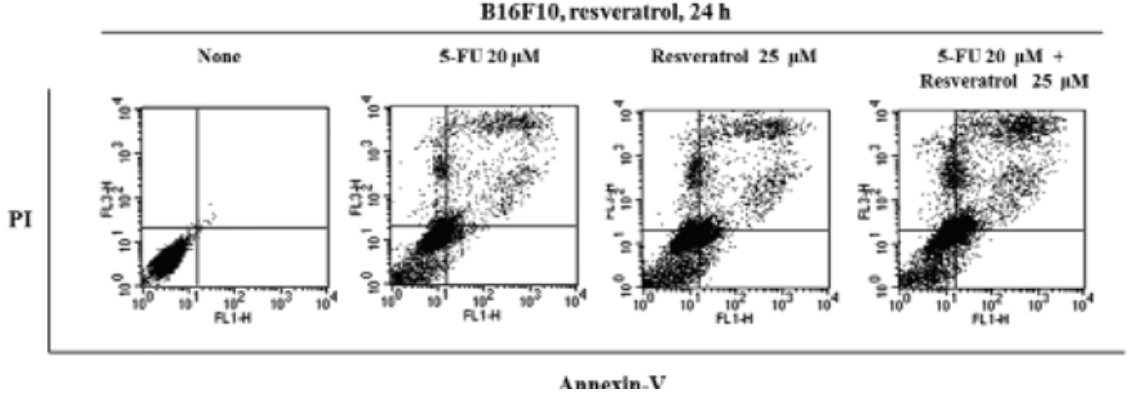

C

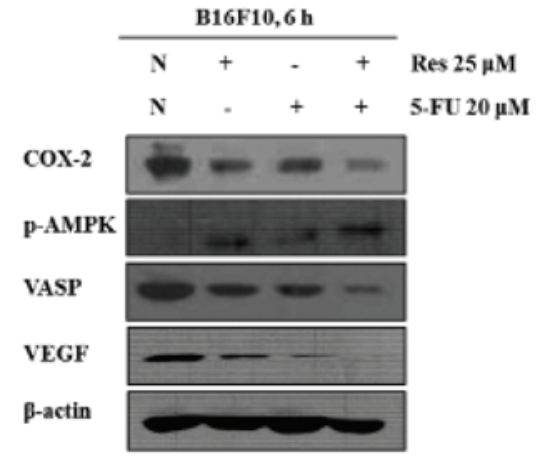

Figure 2. Combination treatment with Res and 5-FU suppresses cell proliferation and induces apoptosis. (A) Cells were treated with 5-FU (20 $\mu \mathrm{M})$, Res $(25 \mu \mathrm{M})$ or co-treated with Res and 5-FU for $24 \mathrm{~h}$ and cell viability was measured using an MTT assay. (B) Cells were treated with 5-FU (20 $\mu \mathrm{M})$, Res $(25 \mu \mathrm{M})$ or co-treated with Res and 5-FU for $24 \mathrm{~h}$. Apoptosis was measured by Annexin V-fluorescein isothiocyanate + PI staining. (C) Cells were treated with Res $(25 \mu \mathrm{M})$ and 5-FU $(20 \mu \mathrm{M})$ or Res in combination with 5-FU for $6 \mathrm{~h}$. The expression levels of p-AMPK, COX-2, VEGF, VASP and $\beta$-actin were examined by western blot analysis. Res, resveratrol; FU, fluorouracil; p-AMPK, phosphorylated AMP-activated protein kinase; COX-2, cyclooxygenase-2; VASP, vasodilator-stimulated phosphoprotein; VEGF, vascular endothelial growth factor; N, none; PI, propidium iodide.

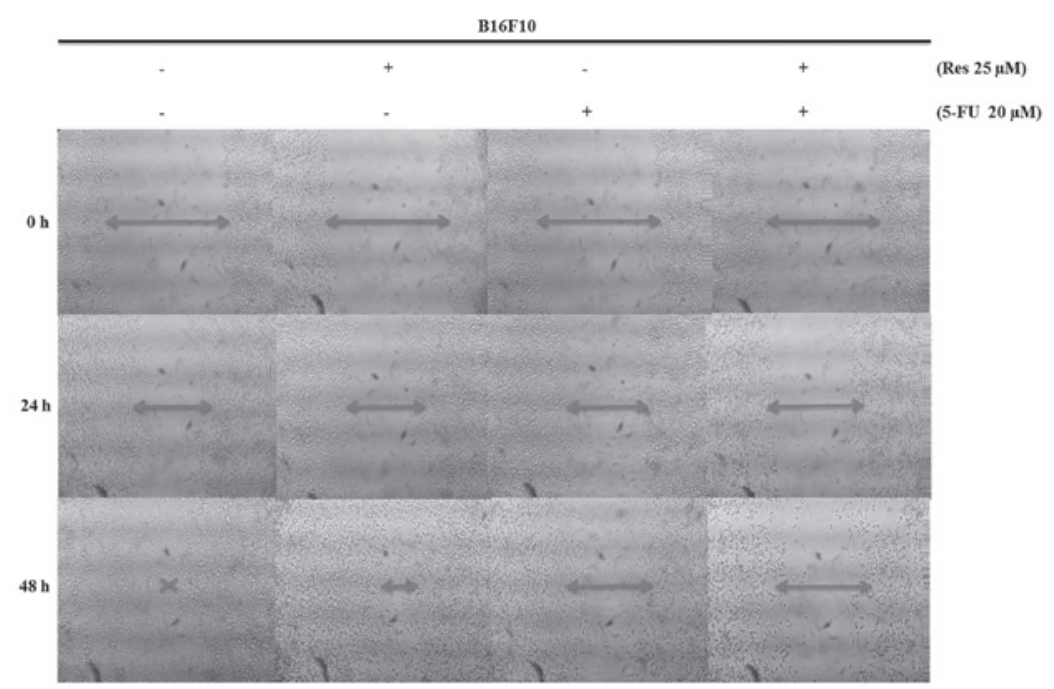

Figure 3. Effect of combined treatment with Res and 5-FU on cell migration. The cells were treated with Res $(25 \mu \mathrm{M})$, 5-FU (20 $\mu \mathrm{M})$ or co-treated with Res $(25 \mu \mathrm{M})$ and 5-FU $(20 \mu \mathrm{M})$ for $24-48 \mathrm{~h}$. Cell motility was measured using a wound healing assay. Representative images of the cell migration were captured (original magnification, x10). Res, resveratrol; Fu, fluorouracil.

the effects of co-treatment with resveratrol and 5-FU on the expression levels of AMPK, COX-2, VASP and VEGF, protein was extracted from the B16 cells treated with resveratrol, 5-FU or a combination of the two. The results demonstrated that phosphorylation of AMPK was increased to a greater extent following co-treatment of resveratrol and 5-FU and that the expression levels of COX-2, VASP and VEGF were decreased more effectively by the co-treatment (Fig. 2C).
Resveratrol and 5-FU inhibit the migration of B16 murine melanoma cells. The present study examined the effects of resveratrol and 5-FU on the migration of B16 cells. The cells were treated with $25 \mu \mathrm{M}$ resveratrol, $20 \mu \mathrm{M} 5$-FU and $25 \mu \mathrm{M}$ resveratrol in combination with $20 \mu \mathrm{M} 5$-FU and the cell migration was measured using a wound healing assay. The untreated B16 cells readily migrated to the wound, whereas cells treated with resveratrol and 5-FU exhibited cell 


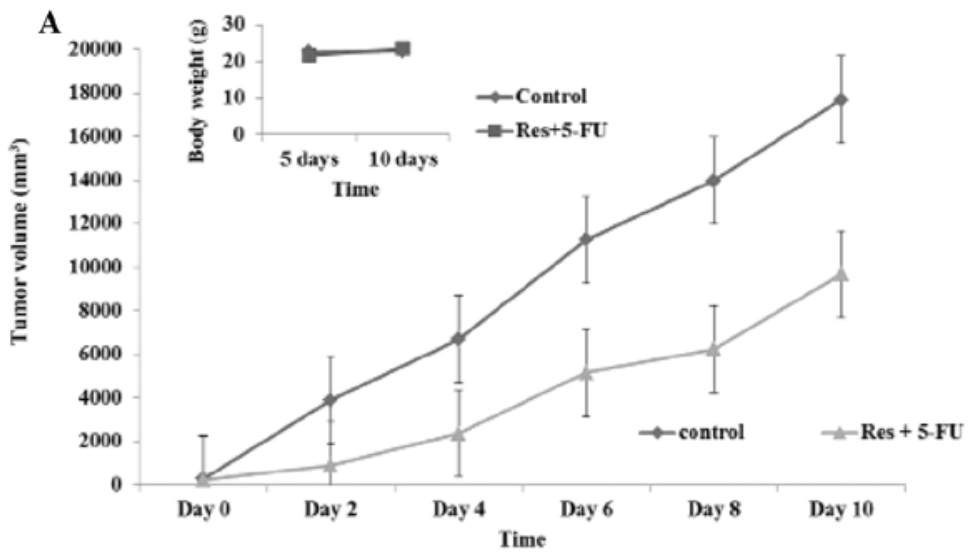

B

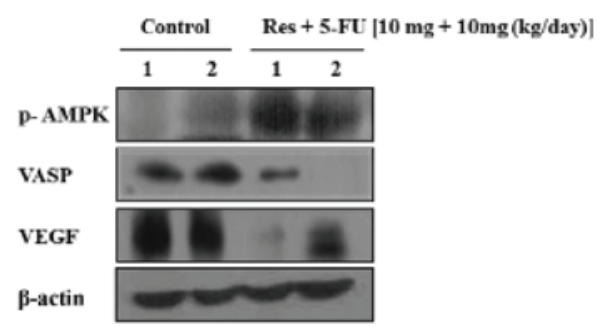

Figure 4. Effect of co-treatment with Res and 5-FU on B16 murine melanoma tumors. B16 murine melanoma cells $\left(2.5 \times 10^{5}\right.$ cells $\left./ 0.1 \mathrm{ml}\right)$ were injected subcutaneously into the left flank of Balb/c nu/nu mice (n=5/group). (A) Tumors were measured each day and tumor volume was calculated. (B) Homogenates of the B16 melanoma tissue from the control and Res $+5-\mathrm{FU}(10 \mathrm{mg} / \mathrm{kg} /$ day of each) mice were used to quantify the levels of p-AMPK, VASP and VEGF by western blot analysis. p-AMPK, phosphorylated AMP-activated protein kinase; VASP, vasodilator-stimulated phosphoprotein; VEGF, vascular endothelial growth factor; Res, resveratrol; FU, fluorouracil.

flattening and the cell migration was inhibited in a dose- and time-dependent manner. The migration of cells treated with a combination of resveratrol and 5-FU was inhibited to a greater extent compared with either drug alone (Fig. 3).

Growth inhibitory effects of combined resveratrol and 5-FU treatment in vivo via regulation of AMPK, VASP and VEGF. To investigate the effect of combined resveratrol and 5-FU treatment in vivo, a B16 xenograft animal model was established. A combination of resveratrol and $5-\mathrm{FU}(10 \mathrm{mg} / \mathrm{kg}$ resveratrol $+10 \mathrm{mg} / \mathrm{kg} 5$-FU/day) was injected intraperitoneally once a day for 10 days, starting one week after the initial injection of B16 cells. Following treatment of the mice with a combination of resveratrol and 5-FU for 10 days, tumor growth decreased compared with the control group, although body weights were unchanged (Fig. 4A). Protein was extracted from the tumors and, as shown in Fig. 4B, the phosphorylation of AMPK was increased by combined resveratrol and 5-FU treatment, while the expression levels of VASP and VEGF were reduced.

Decreased microvessel density in B16 tumors treated with a combination of resveratrol and 5-FU. To examine the effect of combined resveratrol and 5-FU treatment on angiogenesis in the present study, morphometric analysis of immunohistochemical staining for CD31, a marker for microvessel density, was performed. Images of the CD31 staining of microvessels revealed that tumors treated with resveratrol and 5-FU combined had fewer microvessels compared with those in the control group (Fig. 5). These results suggested that combined treatment with resveratrol and 5-FU effectively suppressed angiogenesis.

\section{Discussion}

Angiogenesis, the growth of new vessels from the pre-existing vascular network, is essential for solid tumor growth and metastasis (10) and depends on specific growth factors (2). Therefore, the inhibition of growth factors is widely considered

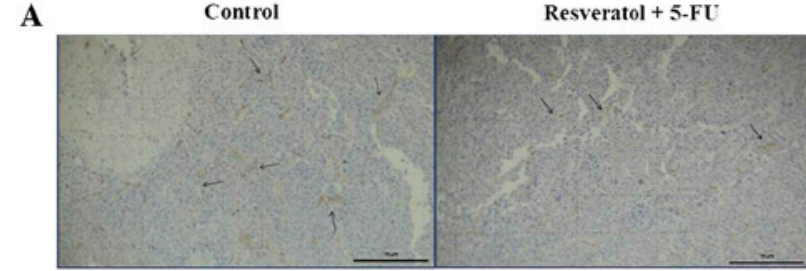

B

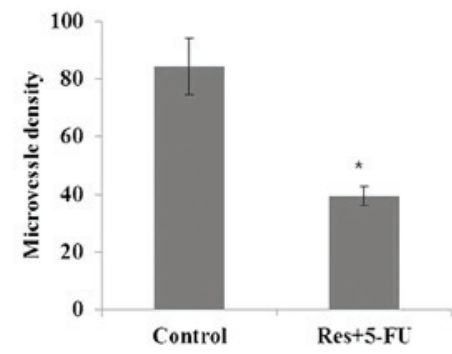

Figure 5. Immunohistochemical staining for microvessels in the melanoma tissues. (A) Microvessel density was observed, as determined by CD31 staining. The brown staining indicates microvascular vessels. The slides were observed under a microscope (magnification, x10) and images were captured. (B) Morphometric analysis indicated the average microvessel density (number of microvessels $/ \mathrm{mm}^{2}$ area). ${ }^{*} \mathrm{P}<0.05$, compared with the control group ( $\mathrm{n}=3$ in each group). Res, resveratrol; FU, fluorouracil.

as one of the most effective strategies for the efficient suppression of tumor growth. A key gene involved in tumor growth is COX-2, which is required for angiogenesis, cell migration and invasion and is overexpressed in various cancer cells (12). A previous study demonstrated that the COX-2-selective inhibitors, JTE-522 and NS-398, significantly reduced tumor mass and vascular density (22). VEGF is another pro-angiogenic factor, which can promote tumor progression, metastasis and cancer cell dissemination (18). In B16F10 melanoma, VEGF inhibition by anti-VEGF agents decreases tumor growth and microvessel density (23). Another protein involved in angiogenesis is VASP, which regulates cell-cell and cell-matrix interactions by regulating actin filament networks and is phosphorylated by AMPK (24). 
5-FU is used to treat various cancer cells; however, several types of tumor have developed resistance to it (8). Therefore, several studies have evaluated novel treatments or 5-FU combination treatments to overcome this drug resistance. Previous studies have demonstrated that co-treatment of cells with genistein and 5-FU reduces the proliferation of colon cancer cells more effectively compared with genistein or 5-FU alone (4), and the co-treatment of colon cancer cells with resveratrol and 5-FU inhibited cell growth more effectively compared with resveratrol or 5-FU alone (25). In the present study, the combination treatment of 5-FU and resveratrol was investigated in order to develop modalities to overcome drug resistance. Resveratrol, a natural polyphenol present in grapes and red wine, has been observed to suppress the proliferation and survival of cancer cells via several mechanisms, including the inhibition of angiogenesis, induction of apoptosis and cell cycle arrest $(1,3,26)$.

The present study demonstrated the synergistic effects of combined resveratrol and 5-FU treatment on B16 cell proliferation and angiogenesis by regulating the expression levels of COX-2, VASP and VEGF in the B16 cells. The combined resveratrol and 5-FU treatment reduced B16 cell proliferation in a dose-dependent manner. Therefore, resveratrol and 5-FU were identified as compounds effectively inhibiting the growth of B16 cells.

The present study also determined whether the resveratrol and 5-FU-induced inhibition of cell proliferation involved changes in COX-2, VEGF, VASP and p-AMPK. Treatment with resveratrol and 5-FU alone significantly reduced the levels of COX-2, VEGF and VASP and increased AMPK. These results indicated that downregulation of COX-2, VEGF and VASP and upregulation of AMPK may have a significant role in the resveratrol- and 5-FU-induced inhibition of B16 cell proliferation.

To examine the effect of combined resveratrol and 5-FU treatment, B16 cells were treated with the two drugs either alone or in combination. The combination of the two drugs inhibited cell proliferation more effectively compared with either drug alone. To further understand whether this inhibition of cell proliferation was due to apoptosis, Annexin V/PI staining was performed. Treatment with resveratrol or 5-FU alone induced apoptosis in the B16 cells; however, the combination of the two was more effective. These results indicated that the inhibition of cell proliferation and the induction of apoptosis was greater when resveratrol and 5-FU were used in combination rather than alone. The present study also investigated whether a combination of resveratrol and 5-FU regulated the expression levels of COX-2, VEGF, VASP and p-AMPK. Resveratrol and 5-FU increased p-AMPK activation and decreased the expression levels of COX-2, VEGF and VASP and, again, this effect was increased when the drugs were used in combination. These results indicated that treatment with a combination of resveratrol and 5-FU had synergistic effects on the induction of apoptosis and on regulation of the expression of COX-2, VEGF, VASP and p-AMPK in the B16 cells.

To evaluate the effect of the combination of resveratrol and 5-FU on cell migration in the present study, a wound healing assay was used. Resveratrol and 5-FU in combination effectively inhibited the migration of B16 cells, which was more marked compared with the effect of either drug alone. In a previous study, resveratrol was found to repress the migration and invasion of LoVo cells by inhibiting the expression levels of VEGF and MMP-9 (27). Furthermore, glioma tissues treated with resveratrol exhibited a reduction in angiogenesis (28). These results indicated that combination treatment with resveratrol and 5-FU suppressed metastasis by inhibiting cell migration.

The present study investigated the synergistic effect of resveratrol and 5-FU on angiogenesis in vitro and demonstrated that the combination of resveratrol and 5-FU was more effective than either drug alone in preventing cell proliferation, migration and apoptosis. In a previous study, the use of resveratrol and 5-FU in combination to treat liver tumors led to more marked inhibition of tumor growth compared with that of either drug alone (10). Furthermore, in an in vivo model of cholangiocarcinoma, co-treatment with resveratrol and 5-FU decreased tumor growth significantly more compared with treatment with either drug alone and a terminal deoxynucleotidyl transferase dUTP nick end labeling assay demonstrated that this combination was also more effective in inducing apoptosis (29). The results of the present study demonstrated that resveratrol or 5-FU alone decreased tumor growth; however, co-treatment with resveratrol and 5-FU was even more effective. However, the mechanisms underlying the enhanced antitumor activity of combined resveratrol and 5-FU treatment remain to be fully elucidated. Therefore, the present study also examined the synergistic effect of combined resveratrol and 5-FU on the regulation of angiogenic factors and B16 tumor growth in vivo.

To assess the effects of co-treatment with resveratrol and 5-FU on B16 tumor growth, mice were treated with resveratrol and 5-FU. Co-treatment with resveratrol and 5-FU reduced tumor growth significantly compared with that in the control group. Changes in the protein expression levels of p-AMPK, VASP and VEGF were also examined and the expression levels of p-AMPK increased, while the expression levels of VASP and VEGF decreased in the mice treated with a combination of resveratrol and 5-FU compared with levels in the control group. In order to evaluate the association between the resveratrol- and 5-FU-mediated inhibition of tumor growth and angiogenesis, immunohistochemical staining for CD31 was used, which specifically stains microvessels. The staining revealed that co-treatment with resveratrol and 5-FU reduced microvessel density compared with that in the control group. These results suggested that the decrease in microvascular vessels in tumor angiogenesis coincided with the decrease in tumor size when using a combination of resveratrol and 5-FU.

In conclusion, the present study indicated that resveratrol and 5-FU have antiproliferative and proapoptotic effects in B16 cells, which are greater when the drugs are used in combination. These effects were mediated, at least in part, by downregulation of the expression levels of COX-2, VEGF and VASP and the resultant inhibition of angiogenesis.

\section{Acknowledgements}

This study was supported by the National Research Foundation of Korea (no. KRF-2012-0021402).

\section{References}

1. Belguendouz L, Fremont L and Linard A: Resveratrol inhibits metal ion-dependent and independent peroxidation of porcine low-density lipoproteins. Biochem Pharmacol 53: 1347-1355, 1997. 
2. Emmett MS, Dewing D and Pritchard-Jones RO: Angiogenesis and melanoma - from basic science to clinical trials. Am J Cancer Res 1: 852-868, 2011.

3. Garvin S, Ollinger K and Dabrosin C: Resveratrol induces apoptosis and inhibits angiogenesiss in human breast cancer xenografts in vivo. Cancer Lett 231: 113-122, 2006.

4. Hwang JT, Ha J and Park OJ: Combination of 5-fluorouracil and genistein induces apoptosis synergistically in chemo-resistant cancer cells through the modulation of AMPK and COX-2 signaling pathways. Biochem Biophys. Res Commun 332 433-440, 2005

5. Cao Z, Fang J, Xia C, Shi X and Jiang BH: trans-3,4,5'-trihydroxystibene inhibits hypoxia-inducible factor 1alpha and vascular endothelial growth factor expression in human ovarian cancer cells. Clin Cancer Res 10: 5253-5263, 2004.

6. Kimura $\mathrm{Y}$ and Okuda $\mathrm{H}$ : Resveratrol isolated from Polygonumcuspidatum root prevents tumor growth and metastasis to lung and tumor-induced neovascularization in Lewis lung carcinoma-bearing mice. J Nutr 131: 1844-1849, 2001.

7. Bråkenhielm E, Cao R and Cao Y: Suppression of angiogenesis, tumor growth, and wound healing by resveratrol, a natural compound in red wine and grapes. FASEB J 15: 1798-1800, 2001

8. Borralho PM, Moreira da Silva IB, Aranha MM, et al: Inhibition of Fas expression by RNAi modulates 5-fluorouracil-inducedapoptosis in HCT116 cells expressing wild-type p53. Biochim Biophys Acta 1772: 40-47, 2007.

9. Ortiz R, Prados J, Melguizo C, et al: 5-Fluorouracil-loaded poly ( $\varepsilon$-caprolactone) nanoparticles combined with phage E gene therapy as a new strategy against colon cancer. Int J Nanomedicine 7: 195-107, 2012.

10. Wu SL, Sun ZJ, Yu L, et al: Effect of resveratrol and in combination with 5-FU on murine liver cancer. World J Gastroenterol 10 3048-3052, 2004.

11. Subbaramaiah K and Dannenberg AJ: Cyclooxygenase 2: a moleculartarget for cancer prevention and treatment. Trends Pharmacol Sci 24: 96-102, 2003.

12. Xiao X, Shi D, Liu L, et al: Quercetin suppresses cyclooxygenase-2 expression and angiogenesis through inactivation of P300 signaling. PLoS One 6: e22934, 2011

13. Greenhough A, Smartt HJ, Moore AE, et al: The COX-2/PGE2 pathway: key roles in the hallmarks of cancer and adaptation to the tumour microenvironment. Carcinogenesis 30: 377-386, 2009.

14. Yao L, Liu F, Hong L, et al: The function and mechanism of COX-2 in angiogenesis of gastric cancer cells. J Exp Clin Cancer Res 30: doi: 10.1186/1756-9966-30-13 2011.

15. Zykova TA, Zhu F, Zhai X, et al: Resveratrol directly targets COX-2 to inhibit carcinogenesis. Mol Carcinog 47: 797-805, 2008 .
16. Kim HN, Kim H, Kong JM, et al: Vitamin C down-regulatesVEGF production in $\mathrm{B} 16 \mathrm{~F} 10$ murine melanoma cells via the suppression of p42/44 MAPK activation. J Cell Biochem 112: 894-901, 2011.

17. Wu G, Luo J, Rana JS, et al: Involvement of COX-2 in VEGF-induced angiogenesis via P38 and JNK pathways in vascular endothelial cells. Cardiovasc Res 69: 512-519, 2006.

18. Kumar B, Chile SA, Ray KB, et al: VEGF-C differentially regulates VEGF-A expression in ocular and cancer cells; promotes angiogenesis via RhoA mediated pathway. Angiogenesis 14: 371-380, 2011.

19. Kim KJ, Li B, Winer J, et al: Inhibition of vascular endothelial growth factor-induced angiogenesis suppresses tumour growth in vivo. Nature 362: 841-844, 1993

20. Price CJ and Brindle NP: Vasodilator-stimulated phosphoprotein is involved in stress-fiber and membrane ruffle formation in endothelial cells. Arterioscler Thromb Vasc Biol 20: 2051-2056, 2000.

21. Philippar U, Roussos ET, Oser M, et al: A Mena invasion isoform potentiates EGF-induced carcinoma cell invasion and metastasis. Dev Cell 15: 813-828, 2008.

22. Amano H, Hayashi I, Endo H, et al: Host prostaglandin E(2)-EP3 signaling regulates tumor-associated angiogenesis and tumor growth. J Exp Med 197: 221-232, 2003.

23. Ghosh S and Maity P: Augmented antitumor effects of combination therapy with VEGF antibody and cisplatin on murine B16F10 melanoma cells. Int Immunopharmacol 7: 1598-1608, 2007.

24. Blume C, Benz PM, Walter U, et al: AMP-activated protein kinase impairs endothelial actin cytoskeleton assembly by phosphorylating vasodilator-stimulated phosphoprotein. J Biol Chem 282: 4601-4612, 2007.

25. Colin D, Gimazane A, Lizard G, et al: Effects of resveratrol analogs on cell cycle progression, cell cycle associated proteins and 5 fluoro-uracil sensitivity in human derived colon cancer cells. Int J Cancer 127: 2780-2788, 2009.

26. Zhang W, Fei Z, Zhen HN, Zhang JN and Zhang X: Resveratrol inhibits cell growth and induces apoptosis of rat C6 glioma cells. J Neurooncol 81: 231-240, 2007.

27. Wu H, Liang X, Fang Y, et al: Resveratrol inhibit hypoxia-induced metastasis potential enhance ment by restricting hypoxia-induced factor-1 alpha expression in colon carcinoma cells. Biomed Pharmacother 62: 613-621, 2008.

28. Chen JC, Chen Y, Lin JH, Wu JM and Tseng SH: Resveratrol suppresses angiogenesis in gliomas: evaluation by color Doppler ultrasound. Anticancer Res 26: 1237-1245, 2006.

29. Frampton GA, Lazcano EA, Li H, Mohamad A and DeMorrow S: Resveratrol enhances the sensitivity of cholangiocarcinoma to chemotherapeutic agents. Lab Invest 90: 1325-1338, 2010. 